\title{
Gedanken Experiment (Thought Experiment) about Gravo-Electric and Gravo-Magnetic Fields, and the Link to Gravitons and Gravitational Waves in the Early Universe
}

\author{
Andrew Walcott Beckwith \\ Physics Department, Chongqing University Huxi Campus, College of Physics, Chongqing University, \\ Chongqing, China \\ Email: Rwill9955b@gmail.com, abeckwith@uh.edu
}

Received 4 November 2015; accepted 24 April 2016; published 27 April 2016

Copyright (C) 2016 by authors and Scientific Research Publishing Inc.

This work is licensed under the Creative Commons Attribution International License (CC BY). http://creativecommons.org/licenses/by/4.0/

(c) (i) Open Access

\begin{abstract}
Our Gedanken experiment is a thought experiment as to what is called gravo-electric and gravomagnetic potentials linked to gravo-electric and gravo-magnetic fields. We examine what Padmanabhan presented in an exercise as of a linkage of electromagnetic fields with Gravitation. The modifications we bring up take the nonrelativistic approximation as the beginning of an order of magnitude estimate as to gravitons, generated electromagnetic fields, and are by definition linked to the total angular momentum of an initial configuration of "particles" of space-time import. The innovation put into Padmanbhan'/s calculation is to for total mass $M$, used, to substitute in $M$ $\mathrm{N}$ (gravitons) times $\mathrm{m}(\mathrm{g})$, where $\mathrm{m}(\mathrm{g})$ is about $10^{\wedge}-62$ grams, as well as specify distances, for the object spinning as being about Planck length in size, give or take a few orders of magnitude. The results are by definition very crude, and do not take into account relativistic effects, but are probably within an order of magnitude important comparison. We conclude with an a comment as to the possibility of an additional polarization as due to a response function of an interferometer to "scalar" polarization as maybe indicate a scalar-tensor gravitational theory as a replacement for General Relativity.
\end{abstract}

\section{Keywords}

Biometric Gravity, Gravo-Electric, Gravo-Magnetic, Tensor-Scalar Gravity Theories, Inflaton 


\section{Introduction}

Reviewing What Was Done by Padmanabhan as Far as

Gravo-Electromagnetic Waves Being Set up So as to Have up for Calculation of Using the Results of Initial Energy as Due to

$$
\delta t \Delta E=\frac{h}{\delta g_{t t}} \equiv \frac{h}{a^{2}(t) \cdot \phi} \Rightarrow \Delta E=\frac{h}{\delta t \cdot \delta g_{t t}} \equiv \frac{h}{\delta t \cdot a^{2}(t) \cdot \phi} \text { and Comparing It to a }
$$

\section{More General Energy Expression Given below}

Our tack is to take what is given by the energy expression from [1], with an minimum energy given as, if $\phi$ is an inflaton, and if $a^{2}(t)$ is the square of the scale factor, and $\delta g_{t t}$ a non-dimensional perturbation of the "time" factor in a geodestic, that then by [1] [2]

$$
\Delta E=\frac{\hbar}{\delta t \cdot \delta g_{t t}} \equiv \frac{\hbar}{\delta t \cdot a^{2}(t) \cdot \phi}
$$

And then what we do is to take the work from [3] to come up with a gravo-electromagnetic frequency which is then set as equal to give an initial import of energy according to a frequency

$$
\begin{aligned}
& \Delta E \doteq \hbar \cdot \omega_{g} \\
& \Rightarrow \omega_{g} \sim \frac{1}{\delta t \cdot \delta g_{t t}} \equiv \frac{1}{\delta t \cdot a^{2}(t) \cdot \phi}
\end{aligned}
$$

This frequency, as isolated in Equation (2) will be compared to the frequency generated by the gravo-electric and gravo-magnetic fields given in the following argument below. It will suggest something about the inflaton, as suggested in the last part of this document.

We will take the square of the frequency given in the 2nd line of Equation (2) and compare it to gravo-electric and gravo-magnetic generated frequency value, as our first principle linkage of electromagnetic waves with gravitons. We should keep in mind that the volume, which is for a complete cosmology is small.

So, let us now come up with a gravo-electric \& gravo-magnetic counterpart to Equation (2) above. To do that, we take an argument given in [3] in pages 278 - 279 of the form, from exercise 6.15 of a magnetic and electric field being generated by a source with arbitrary density, as to have the following two lowest order perturbations, as given in the exercise to be, if $M$ is total mass, $J^{\alpha \beta}$ the angular momentum tensor with also $p^{\alpha}(y)$ a momentum density defined by $p^{\alpha}(y)=\rho(y) \cdot u^{\alpha}(y)$, to yield the gravo-electric tensor $\Phi_{g}(x)$, and gravo-magnetic potential $A_{g}(x)$. Then, we shall reference the potential's given below. Note, that we are referring to a volume, $V$, which will be for the entire spatial domain of the universe but that our $V$ volume is in early universe conditions of Planckian size dimensions. The author thanks the referee for the necessity of making this point obvious as how to properly interpret Equation (1) given above. Here, $G$ is the usual gravitational "constant", $c$ the speed of light, and what is called $x$ is the spatial dimensions. $J^{\alpha \beta}$ as the angular momentum tensor should be thought of in terms of, in this case, as having a relationship to electromagnetics, as given in [4] below whereas

$$
\begin{aligned}
& \bar{h}^{0 \alpha}=-\frac{2 G}{c^{3}|x|} x_{\beta} J^{\alpha \beta}+\vartheta\left(\frac{1}{|x|^{3}}\right) \\
& M=\int_{V} \rho(y) \cdot \mathrm{d}^{3} y \\
& J^{\alpha \beta}=\int_{V}\left[y^{\alpha} p^{\beta}(y)-y^{\beta} p^{\alpha}(y)\right] \cdot \mathrm{d}^{3} y \\
& \Leftrightarrow \Phi_{g}(x)=-\frac{G M}{|x|} \\
& A_{g}(x)=-\frac{G M}{c^{2}|x|^{3}}(S \times x)
\end{aligned}
$$


we are taking Equation (1) as formulated from equations from pages 278-279 of the text as given in reference [3]

Then again by [3], and exercise 6.15, page 279, the following gravo-electric and gravo-magnetic fields appear

$$
\begin{aligned}
& E_{g}(x)=-\frac{G M}{|x|^{2}} \hat{x} \\
& B_{g}(x)=\frac{G}{c^{2}|x|^{3}}(S-3(S \cdot \hat{x}) \hat{x})
\end{aligned}
$$

Here, $\hat{x}$ is a unit vector in the radial direction, and $S$ an angular velocity, which will then lead to the following angular frequency, that by [3], and exercise 6.15 , page 279

$$
\omega^{2}=\frac{G \cdot M}{r^{3}} \mp \frac{2 \cdot G \cdot S}{c^{2} r^{4}} \sqrt{\frac{G M}{r}}
$$

From here, we will proceed to modify $M$, and $S$ by gravitational physics.

\section{Modify $M$, and $S$ by Gravitational Physics}

What we are going to do, is the restrict $M$ to the case of heavy gravity in the Planckian regime, call $G$ the usual gravitational physics variable, and define $S$, as following for $M$ and $S$. $N$ being the number of initial gravitons, and a radii as Planck length $\times 10^{\aleph}$, with $\aleph>0$, so up to a good approximation

$$
\begin{aligned}
M & \approx N_{\text {gravitons }} \cdot m_{\text {graviton }} \\
S & \approx \sum_{j=1}^{N_{\text {gravitons }}}\left(R_{j} \times m_{j} v_{j}(\text { velocity })\right) \\
& \approx N_{\text {gravitons }} \times\left(l_{\text {Planck }} \times 10^{\aleph}\right) \cdot m_{\text {graviton }}
\end{aligned}
$$

Then the maximum initial value of the angular frequency of Equation (5) is

$$
\omega^{2}=\frac{G \cdot N_{\text {gravitons }} \cdot m_{\text {graviton }}}{r^{3}} \mp \frac{2 \cdot G \cdot N_{\text {gravitons }} \times\left(l_{\text {Planck }} \times 10^{\aleph}\right) \cdot m_{\text {graviton }}}{c^{2} r^{4}} \sqrt{\frac{G \cdot N_{\text {gravitons }} \cdot m_{\text {graviton }}}{r}}
$$

\section{Modify $M$, and $S$ by Gravitational Physics with Numerical Inputs into Equation (7) for Frequency}

$N_{\text {gravitons }} \approx 10^{37}$, due to and a rest massive graviton mass of about $10^{\wedge}-62$ grams, due to [5] plus a radial distance $r$ from the source of the graviton production would lead to relic gravitational waves reduced dramatically from the beginning radii presumably about 1 meter, to the present radii of the universe, presumably of the value of about $4.4 \times 10^{26}$ meters .

If so, then, the energy would be represented, if $\lambda_{\text {graviton }}=\frac{2 \pi v(\text { velocity })_{\text {graviton }}}{\omega_{\text {graviton }}}$ and we have by [6]

$$
\left(\frac{v_{\text {graviton }}}{c}\right)^{2}=1-\frac{m_{\text {graviton }}^{2} c^{4}}{E_{\text {graviton }}^{2}}
$$

Then from [4] [6] we have for gravitons an energy value of about, if $m$ is the mass of a "massive" graviton, using in this case the relativistic formula as given in [2] [6], to approximate to first order

$$
E \sim 2 \pi \hbar \cdot \frac{c}{2 \lambda}\left(1 \pm \sqrt{1+\frac{4 m^{2} c^{4}}{\left(\frac{2 \pi \hbar c}{\lambda}\right)^{2}}}\right) \sim \frac{\pi \hbar c}{\lambda}\left(2+\frac{2 m^{2} \lambda^{2} c^{4}}{(2 \pi \hbar \cdot c)^{2}}\right)
$$

Compare this value of energy, by making the following scaling, namely equate Equation (5) and Equation (9) 
such that

$$
\omega^{2}=\frac{G \cdot N_{\text {gravitons }} \cdot m_{\text {graviton }}}{r^{3}} \mp \frac{2 \cdot G \cdot N_{\text {gravitons }} \times\left(l_{\text {Planck }} \times 10^{\aleph}\right) \cdot m_{\text {graviton }}}{c^{2} r^{4}}
$$

Which is then compared with and is implying a frequency squared value of [2]

$$
\omega^{2} \approx\left(\frac{2 \pi c}{\lambda}\right)^{2}\left(1+\frac{m_{\text {gravitons }}^{2} \lambda^{2} c^{4}}{(2 \pi \hbar \cdot c)^{2}}\right)^{2}
$$

To get to the present value of what the relic wavelengthfor produced gravitons initially would be, take in the upper value of the $\lambda$ given in Equation (11) that $m_{\text {graviton }} \approx 10^{-62}$ grams, as in [5] would be if $r$ in Equation (10) is $4.4 \times 10^{26}$ meters

$$
\lambda \approx 10^{9}-10^{10} \text { meters (today) }
$$

Whereas if $r$ in Equation (10) is significantly less than 1 meter, emergent radiation would be

$$
\lambda \approx 10^{-29}-10^{-30} \text { meters (initially) }
$$

Based upon Equation (12) and Equation (13)

$$
\begin{aligned}
& \omega(\text { Initial }) \approx 10^{30}(\text { or }- \text { more }) \text { Hertz } \\
& \omega(\text { Today }) \approx 10^{-10}(\text { or }- \text { less }) \text { Hertz }
\end{aligned}
$$

This is using extremely rough estimates.

\section{Considerations as to Bicep 2, the Matter of Scalar-Tensor Polarizations as an Alternative to General Relativity and Alternate Gravitational Theories and Experimental Tests of General Relativity via Inteferometric Methods}

From [7] we have the following to consider, namely trying to determine restraints upon the nature of gravity, i.e. is it consistent with General relativity or do we have an alternative situation as given in the following quote. We hope that getting a consistent model of inflaton physics will help clarify the following alternatives:

Quote

This fact rules out the possibility of treating gravitation like other quantum theories, and precludes the unification of gravity with other interactions. At the present time, it is not possible to realize a consistent Quantum Gravity Theory which leads to the unification of gravitation with the other forces. On the other hand, one can define Extended Theories of Gravity those semiclassical theories where the Lagrangian is modified, in respect to the standard Einstein-Hilbert gravitational Lagrangian, adding high-order terms in the curvature invariants (terms like R2, etc...) or terms with scalar fields non minimally coupled to geometry (terms like $\varphi 2 R$ )

End of quote

We claim that the strength of the inflaton term, as we will give in Equation (15) may allow us to determine if we have to use a semi classical set of terms which add more terms to the space curvature of early universe Planckian physics space time geometry. We also though have to temper this quest in requiring that the following holds as given in [8], namely:

Quote

Recent data from Planck matches well with the minimal $\Lambda$ CDM model. A likelihood analysis using Planck, WMAP and a selection of high resolution experiments (highL), tensor to scalar ratio $r 0.002$ is found to be $<0.11$ when $\mathrm{dns} / \mathrm{dlnk}=0$.

End of quote

Our inflaton, which is given in Equation (15) must be made consistent with the requirements of a low scalar to tensor ratio, and this requires exquisite fine tuning of inputs into the inflaton, which also should be made consistent with answers to [7]-[9].

We find that the resulting inflaton measurement which is the conclusion of our document, with the following, 
namely assuming that $a$ (initial-start $) \sim 10^{-55}$, and which may involve [10] directly. I.e. is our inflaton consistent with just two standard polarizations, or is there a third polarization necessary so that the following inflaton forms? If there is not a response function of an interferometer to an additional "scalar" polarization we define, we stick to GR, whereas though if Equation (15) necessitates an additional polarization, we are looking at a scalar-tensor gravitational theory. Needless to say we will require careful analysis of

$$
\begin{aligned}
& \delta t \Delta E=\frac{\hbar}{\delta g_{t t}} \equiv \frac{\hbar}{a^{2}(t) \cdot \phi} \\
& \Leftrightarrow \phi(\text { initial }) \approx \frac{1}{t(\text { Planck })} \cdot \frac{1}{\left[a(\text { initial }- \text { start }) \sim 10^{-55}\right]^{2} \cdot\left[\omega(\text { initial }) \approx 10^{30}(\text { or }- \text { more }) \text { Hertz }\right]}
\end{aligned}
$$

This enormous value for the inflaton, initially, needs to be examined further. As given in references [1] [2] as to further prospects. It further should be linked to Corda's pioneering work with "gravity's breath", i.e. traces of the inflaton as given by [10] [11] and is the justification of Equation (15) above. We can use this to determine what to make of the stochastic background of pre space time physics.

\section{Avoiding the Bicep 2 Mistake: What We Can Do with Equation (15)?}

Following [9] what we are doing is examining the stochastic regime of space-time where the following holds:

Quote

Omni-directional gravitational wave background radiation can arise from fundamental processes in the early Universe, or from the superposition of a large number of signals with a point-like origin. Examples of the former include parametric amplification of gravitational vacuum fluctuations during the inflationary era, termination of inflation through axion decay or resonant preheating, Pre-Big Bang models inspired by string theory, and phase transitions in the early Universe; the observation of a primordial background will give access to energy scales of 10 to the 9 power, up to 10 to the 10 power $\mathrm{GeV}$, well beyond the reach of particle accelerators on Earth.

End of quote

Needless to say though, we need above all to avoid getting many multiple stochastic signals, in what we process for primordial gravitational waves, and to use, instead tests to avoid getting dust signals which are what doomed Bicep 2, i.e. as is made very clear in [12] [13]. In all, what we are doing is consistent with the requirements given in the Authors other article, [14], as is given in the following quote:

Quote

The main agenda will be in utilization of Equation (27) to help nail down a range of admissible frequencies which will be to avoid [11]-[14] conflating the frequencies of collected gravitational wave signals from relic cosmological conditions (or would be signals) with those connected with dust generated gravitational wave signals, especially from dust conflated with Galaxy formation in the early universe. More than anything else, we need to find likely narrow (?) Frequency ranges, which will be commensurate with Equation (27), and to use advanced detector technology. Of course such a search will be hard. But it also will be a way, with due diligence as to answer questions raised by the Author in [14]. In doing so, the relative flatness of the early universe and its departure from curved space conditions will be a great way to answer the suppositions raised in [9] [10] as well.

End of quote

Understanding inflaton physics properly will also give credence to considerations given in [1] below as to the degree of flatness or lack of, in the early Universe.

\section{Acknowledgements}

This work is supported in part by National Nature Science Foundation of China grant No. 11375279.

\section{References}

[1] Beckwith, A. (2015) Gedanken Experiment for Degree of Flatness, or Lack of, in Early Universe Conditions. http://vixra.org/pdf/1510.0108v4.pdf

[2] Giovannini, M. (2008) A Primer on the Physics of the Cosmic Microwave Background. World Scientific, Singapore.

[3] Padmanabhan, T. (2010) Gravitation, Foundations and Frontiers. Cambridge University Press, New York. 
http://dx.doi.org/10.1017/CBO9780511807787

[4] Leader, E. The EM Tensor and Its Relation to Angular Momentum. http://www.int.washington.edu/talks/WorkShops/int_12_49W/People/Leader_E/Leader1.pdf

[5] Goldhaber, A. and Nieto, M. (1954) Mass of the Graviton. PRD Volume 9, Number 4.

[6] Will, C. The Confrontation between General Relativity and Experiment, Living Reviews in Relativity. http://relativity.livingreviews.org/Articles/lrr-2006-3/

[7] Corda, C. (2009) Interferometric Detection of Gravitational Waves: The Definitive Test for General Relativity. International Journal of Modern Physics D, 18, 2275-2282. http://arxiv.org/abs/0905.2502 http://dx.doi.org/10.1142/S0218271809015904

[8] Das, S., Mukherjee, S. and Souradeep, T. (2015) "Revised Cosmological Parameters after BICEP 2 and BOSS. JCAP 02 (2015) 016. http://arxiv.org/abs/1406.0857

[9] Van Den Broeck, C. (2015) Gravitational Wave Searches with Advanced LIGO and Advanced Virgo. 50th Rencontres de Moriond, Gravitation: 100 Years after GR, ARISF. http://arxiv.org/pdf/1505.04621v1.pdf

[10] Corda, C. (2007) A Longitudinal Component in Massive Gravitational Waves Arising from a Bimetric Theory of Gravity. Astroparticle Physics, 28, 247-250. http://arxiv.org/abs/0811.0985 http://dx.doi.org/10.1016/j.astropartphys.2007.05.009

[11] Corda, C. (2012) Primordial Gravity’s Breath. EJTP, 9, 1-10. http://arxiv.org/pdf/1110.1772

[12] Cowen, R. Gravitational Waves Discovery Now Officially Dead; Combined Data from South Pole Experiment BICEP2 and Planck Probe Point to Galactic Dust as Confounding Signal. http://www.nature.com/news/gravitational-waves-discovery-now-officially-dead-1.16830

[13] Cowen, R. Full-Galaxy Dust Map Muddles Search for Gravitational Waves. http://www.nature.com/news/full-galaxy-dust-map-muddles-search-for-gravitational-waves-1.15975

[14] Beckwith, A. Non Linear Electrodynamics Contributing to a Minimum Vacuum Energy (“Cosmological Constant”) Allowed in Early Universe cosmology. JHEPGC. 